\title{
Primary mucinous adenocarcinoma of the renal pelvis misdiagnosed as calculous pyonephrosis: a case report and literature review
}

\author{
Huihuang $\mathrm{Li}^{1 \#}$, Fujuan Xie ${ }^{2 \#}$, Cheng Zhao ${ }^{1}$, Zhenglin $\mathrm{Yi}^{1}$, Jinbo Chen ${ }^{1}$, Xiongbing Z ${ }^{1}$ \\ ${ }^{1}$ Department of Urology, ${ }^{2}$ Department of Surgery, Xiangya Hospital, Central South University, Changsha 410008, China \\ "These authors contributed equally to the work and are co-first authors. \\ Correspondence to: Prof. Xiongbing Zu; Jinbo Chen. Department of Urology, Xiangya Hospital, Central South University, No. 87 Xiangya Road, \\ Changsha 410008, China. Email: zuxbxyyy@126.com; chenjinbo1989@yahoo.com.
}

\begin{abstract}
Primary mucinous adenocarcinoma of the renal pelvis is a rare malignant disease that is difficult to diagnose preoperatively. There are still no characteristic symptoms, radiological features, or standard treatment for this tumor with only $\sim 100$ cases reported. The prognosis is poor. We report a case of a 66-year-old man who presented with a 2 -month history of fever and right waist pain. He was misdiagnosed with calculous pyonephrosis and underwent percutaneous nephrostomy (PCN) at a local hospital. Gelatinous material was drained via a PCN catheter. He was then transferred to our hospital. He had elevated CEA and CA19-9. We performed an open radical nephrectomy and found polypoid, gelatinous material and stones filling the renal pelvis. He was diagnosed with primary mucinous adenocarcinoma of the renal pelvis by pathology. He refused adjuvant chemotherapy and there was no sign of recurrence after one year of followup. By assessing a literature review of all of the cases reported since 2000, we recommend that careful history taking, serum tumor markers, and CT scans may improve the diagnostic accuracy rates and radical nephrectomy with total ureterectomy accompanied by adjuvant therapy may improve the prognosis.
\end{abstract}

Keywords: Adenocarcinoma; mucinous; kidney pelvis; biomarkers, tumor; pyonephrosis; case reports

Submitted Nov 07, 2019. Accepted for publication Dec 23, 2019.

doi: $10.21037 /$ tau.2019.12.38

View this article at: http://dx.doi.org/10.21037/tau.2019.12.38

\section{Introduction}

Transitional cell carcinoma and squamous cell carcinoma are the most common epithelial-origin malignancies arising from the renal pelvis, accounting for $85-90 \%$ and $10-15 \%$ of cases, respectively (1). Adenocarcinomas of the renal pelvis accounts for less than $1 \%$ of cases and can be subclassified as tubulovillous $(71.5 \%)$, mucinous $(21.5 \%)$, and papillary non-intestinal $(7.0 \%)(1,2)$. Hasebe et al. first reported primary mucinous adenocarcinoma of the renal pelvis in 1960 (3). It remains an especially rare disease with fewer than 100 cases reported (4). It is difficult to diagnose preoperatively without characteristic symptoms or radiological features. Also, because of its' rarity, no standard treatment has been proposed (1). Herein, we present a case of mucinous adenocarcinoma arising from the renal pelvis in accordance with the CARE Guidelines and conduct a literature review including all of the cases reported since 2000 .

\section{Case presentation}

A 66-year-old man presented with a 2-month history of fever and right waist pain without nausea, vomiting, hematuria, or pyuria. His past medical history also included a diagnosis of hepatitis B. He was admitted to a local hospital. A computed tomography scan showed multiple renal pelvic calculi, stenosis of the ureter, and severe hydronephrosis with cortical thinning (Figure 1A,B). He was diagnosed with calculous pyonephrosis and underwent percutaneous nephrostomy (PCN). A considerable amount 

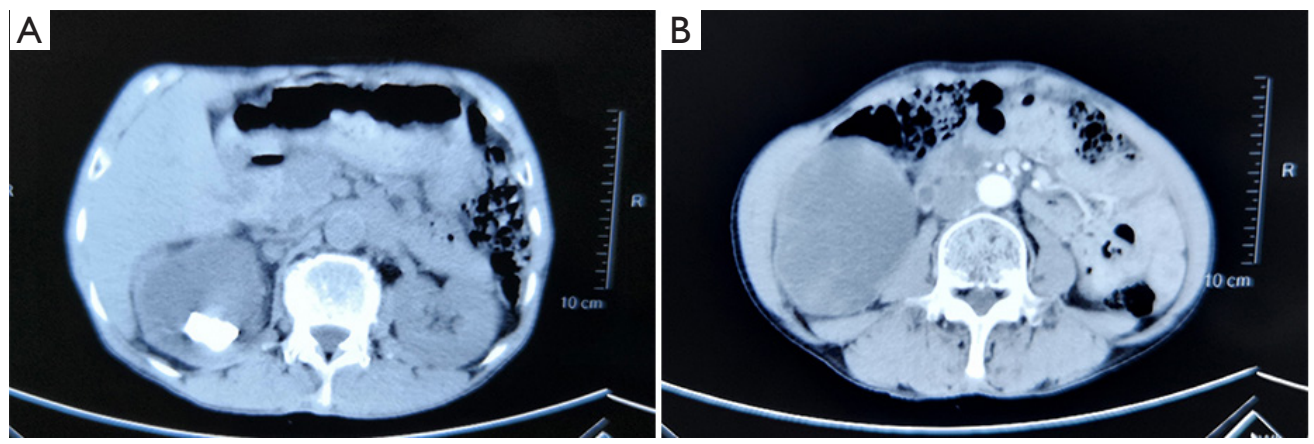

Figure 1 Abdominal computed tomography scan. (A) Multiple renal pelvic calculi and severe hydronephrosis with cortical thinning; (B) stenosis of ureter and severe hydronephrosis with cortical thinning.
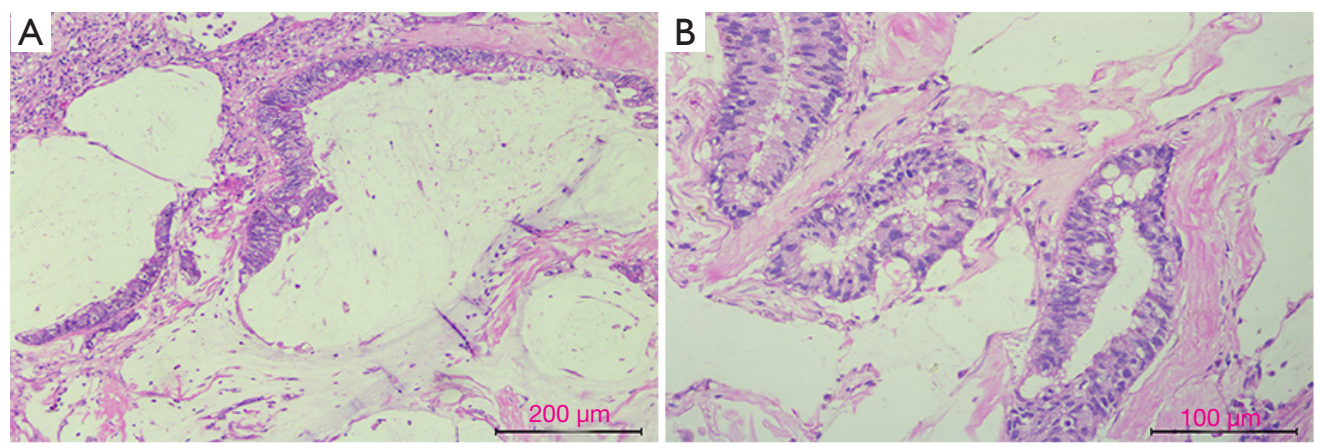

Figure 2 Histological findings of the tumor. (A) (100x) and (B) (200x) hematoxylin and eosin staining indicating intestinal metaplasia and glandular acini.

of gelatinous material was drained via a PCN catheter without urine. The catheter was blocked the second day and PCN was performed again. However, these methods could not control the patient's fever and he was transferred to our hospital. Physical examination was generally normal except for percussion tenderness on the right kidney region. Laboratory tests demonstrated elevated red $(20 / \mu \mathrm{L})$ and white blood cells $(200 / \mu \mathrm{L})$ in the urinalysis, a decreased red blood cell count $\left(4.29 \times 10^{12} / \mathrm{L}\right)$ and hemoglobin concentration $(118 \mathrm{~g} / \mathrm{L})$, and elevated CEA $(7.89 \mathrm{ng} / \mathrm{mL})$ and CA19-9 (5.79 $\mathrm{ng} / \mathrm{mL})$. HBsAg was positive. Liver function, renal function, coagulation function, and stool routine examination were generally normal. Chest computed tomography scan showed an old tuberculosis scar on the right lung. We suspected that he had gastrointestinal cancer and performed an upper gastrointestinal endoscopy and a colonoscopy. However, nothing abnormal was found on the gastric or colonic mucosa, and the gelatinous material collected from the PCN catheter indicated no malignancy.
We diagnosed the patient with calculous pyonephrosis and malignant tumor to be excluded. We then performed an open radical nephrectomy. His kidney was markedly enlarged with a thinning renal cortex. There was an unintentional spillage of gelatinous material because of the two PCN procedures. Therefore, we only performed a nephrectomy without total ureterectomy. After opening the kidney, there were polypoid, gelatinous material and stones filling the renal pelvis. Histologically, the tumor was detected intestinal metaplasia and glandular acini with multiple extracellular mucin (Figure 2). Immunohistochemistry revealed that CDX2, CEA, Villin and ki67 (60\%) were positive (Figure 3) and CA125, MUC6, CK7, CD20, GATA3, S100P, and SATB2 were negative. The histologic diagnosis of primary mucinous adenocarcinoma of the renal pelvis was conducted. This patient was advised to undergo adjuvant chemotherapy because of the spillage of gelatinous material during surgery, but he refused. After one year of follow-up, the patient reported no discomfort, and a computed tomography scan 

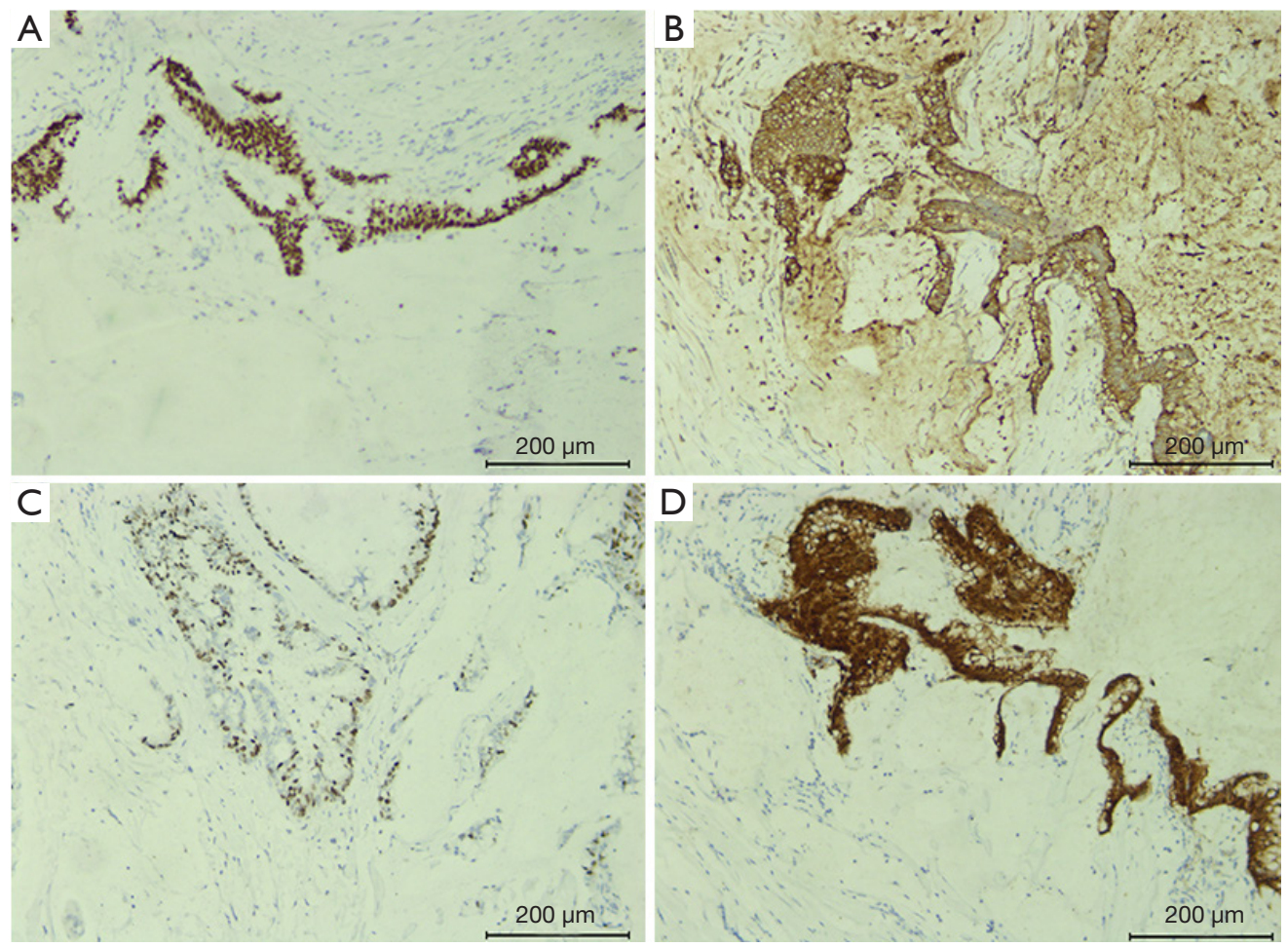

Figure 3 Immunohistochemistry (A) positive for CDX2 (100x); (B) positive for CEA (100x); (C) positive for Villin (100x); (D) positive for ki67 (60\%) (100x).

indicated no sign of recurrence. Examination of the serum tumor markers indicated that CEA was $3.57 \mathrm{ng} / \mathrm{mL}$. Figure 4 depicts a timeline of the diagnosis, interventions, and outcomes.

\section{Discussion}

Mucinous adenocarcinoma, generally seen in the colorectal and ovarian regions, is characterized by abundant mucous secretion comprising more than $50 \%$ of the tumor volume $(5,6)$. Primary mucinous adenocarcinoma of the renal pelvis is an especially rare disease with fewer than 100 cases reported to date. It is poorly recognized and can be misdiagnosed as calculous pyonephrosis. As in the present case, PCN can result in iatrogenic tumor-cell spreading and local seeding. PCN can also increase the difficulty of radical nephrectomy. These can contribute to poor patient prognosis. Although we are not the first group to report this disease, our case should remind surgeons to be cautious about the possibility of malignancy before conducting PCN on patients with pyonephrosis, especially those with elevated serum tumor markers.
We conducted a literature search of PubMed and Embase using MeSH terms 'Adenocarcinoma, Mucinous' and 'Kidney Pelvis'. We included all of the cases reported in English since 2000 (Table 1). According to our literature review, most of the cases were from Asian countries (83.3\%), especially India (33.3\%). More male patients were reported than females $(63.33 \%$ vs. $33.33 \%)$. In mucinous ovarian cancer, the only clinical risk factor is tobacco smoking (6). The prevalence of male smoking may cause the higher rate in males. No characteristic symptoms were reported in previous cases. As in our case, the patient presented with intermittent fever and right waist pain. Our literature review found that there were $19 / 30$ of patients presenting with a flank pain or discomfort, 7/30 of patients presenting with abdominal mass, and 5/30 of patients presenting with hematuria. These were consistent with the classic triad of renal cancer, indicating long-standing or latestage disease. It is reported that the long-standing obstruction, chronic irritation, and infection can contribute to glandular metaplasia of the urothelium, dysplasia, and adenocarcinoma (8). However, some researchers thought that suspecting or diagnosing this tumor via history taking 

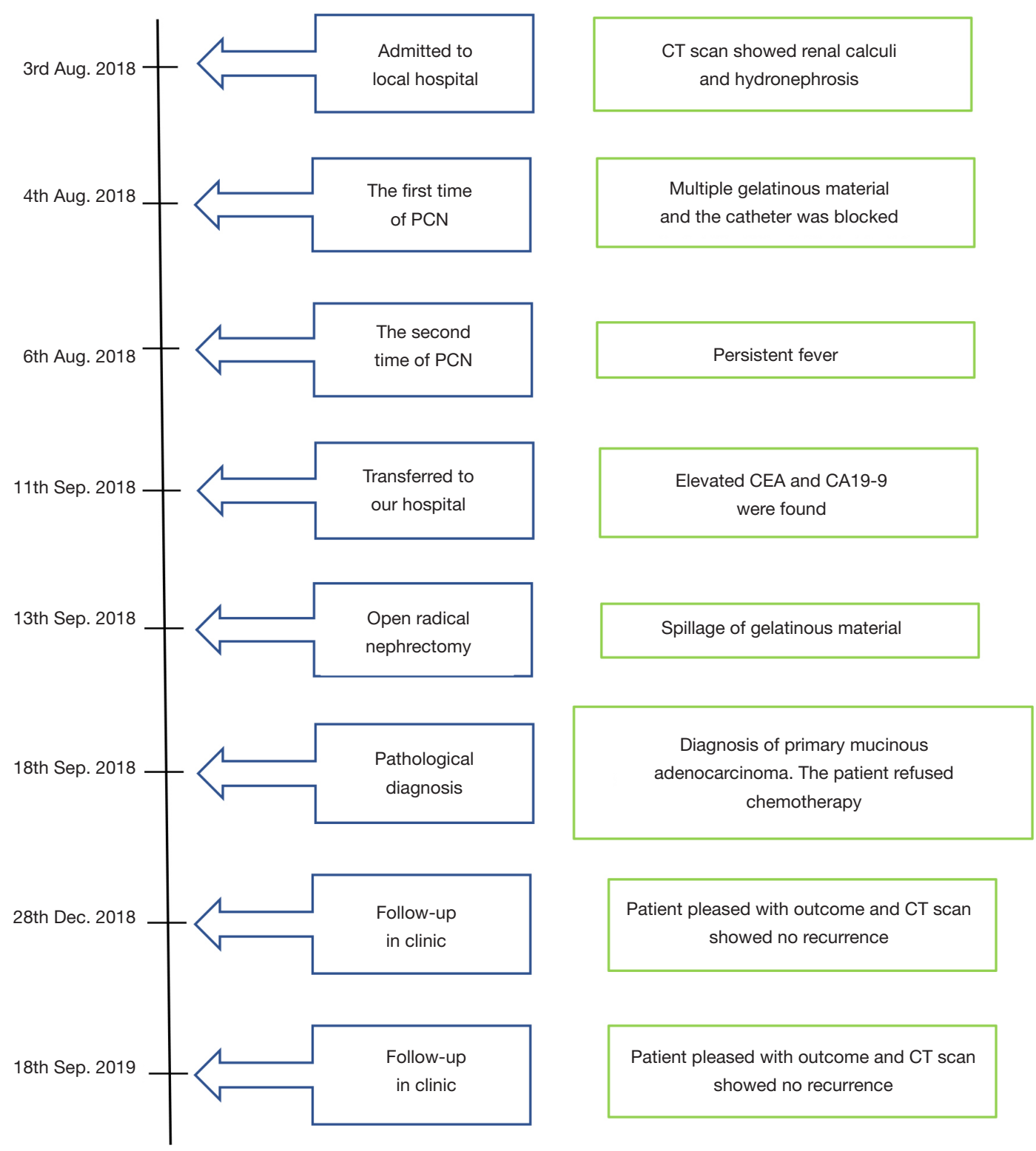

Patient pleased with outcome and CT scan showed no recurrence

Figure 4 Timeline of interventions and outcomes.

and physical examination were impossible (1). Through this case and literature review, we believe careful history taking and physical examination could indicate long-standing disease and contribute to the preoperative diagnosis.

There were no characteristic radiological features of primary mucinous adenocarcinoma (18). Our patient presented with multiple renal pelvic calculi, stenosis of the ureter, and severe hydronephrosis with cortical thinning. These features made it easy to diagnose calculous pyonephrosis. In our literature review, there were $16 / 30$ of patients presenting with hydronephrosis, 9/30 of patients presenting with calculi, 10/30 of patients presenting with pyonephrosis, $9 / 30$ of patients presenting with a mass or tumor, and a few patients with pelvis ureteric junction obstruction or stenosis. All of these features except for a mass do not indicate a malignancy. According to most of the reported cases, hydronephrosis and non-functional kidney caused by renal calculi were diagnosed by computed tomography. Only after pathologic analysis, could primary mucinous 


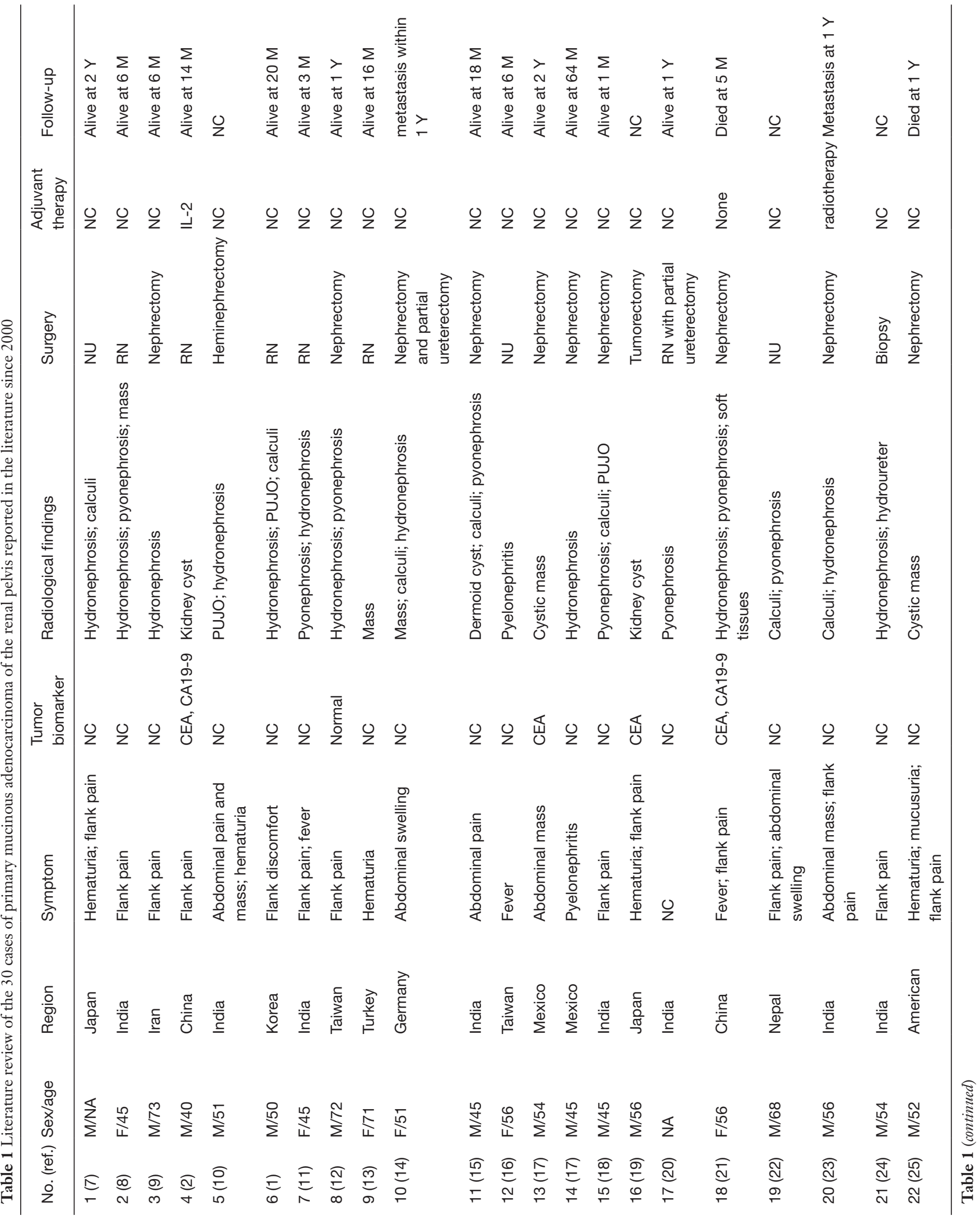




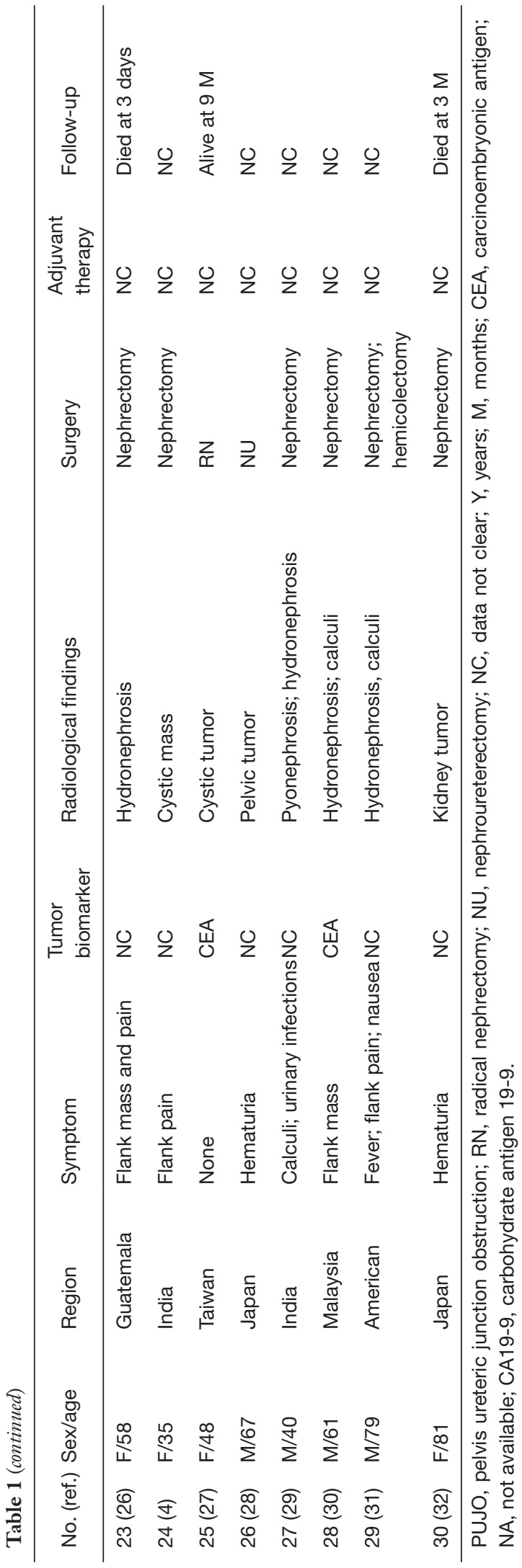

adenocarcinomas of the renal pelvis be diagnosed (12). However, there were a few cases reporting that primary mucinous adenocarcinomas of the renal pelvis could be accompanied by elevated CEA or CA19-9 (2). Our literature review found that $20 \%$ of the patients had elevated CEA or CA19-9. We hypothesize that serum tumor markers accompanied by CT scans an increase the diagnostic accuracy rates.

No standard surgical procedures have been proposed for this adenocarcinoma (2). As our literature review indicated, most of the patients underwent a nephrectomy without total ureterectomy because there were no preoperative measures to detect this tumor (14). However, the standard treatment for pelvis tumors is nephroureterectomy with a bladder cuff (28). Moreover, few of the patients received adjuvant therapy. Only Lai et al. (2) and Raphael et al. (23) reported that interleukin-2 and radiotherapy were administered after surgery, respectively. The prognosis of primary adenocarcinoma of the renal pelvis is generally poor and most patients die during 2-5 years of follow-up (21). It is reported that chemotherapy, radiotherapy, and chemoradiotherapy should be recommended for mucinous colorectal cancer and mucinous ovarian carcinoma $(5,6)$. We recommend adjuvant therapy such as chemotherapy after nephroureterectomy with a bladder cuff to improve the prognosis.

In conclusion, primary mucinous adenocarcinoma of the renal pelvis is especially rare without characteristic radiological features and standard treatment. Based on our literature review, careful history taking and radiological examination accompanied by serum tumor markers may improve the diagnostic accuracy rates. Adjuvant therapy is recommended after nephroureterectomy for better survival outcomes.

\section{Acknowledgments}

Funding: This work was supported by the National Natural Science Foundation of China $(81572523,81700665)$.

\section{Footnote}

Conflicts of Interest: All authors have completed the ICMJE uniform disclosure form (available at http://dx.doi. org/10.21037/tau.2019.12.38). XZ serves as an unpaid editorial board member of Translational Andrology and Urology from Mar 2019 to Feb 2021. The other authors have no conflicts of interest to declare. 
Ethical Statement: The authors are accountable for all aspects of the work in ensuring that questions related to the accuracy or integrity of any part of the work are appropriately investigated and resolved. Written informed consent was obtained from the patient for publication of this manuscript and any accompanying images.

Open Access Statement: This is an Open Access article distributed in accordance with the Creative Commons Attribution-NonCommercial-NoDerivs 4.0 International License (CC BY-NC-ND 4.0), which permits the noncommercial replication and distribution of the article with the strict proviso that no changes or edits are made and the original work is properly cited (including links to both the formal publication through the relevant DOI and the license). See: https://creativecommons.org/licenses/by-nc$\mathrm{nd} / 4.0 \%$.

\section{References}

1. Han DS, Yuk SM, Youn CS, et al. Primary mucinous cystadenocarcinoma of the renal pelvis misdiagnosed as ureteropelvic junction stenosis with renal pelvis stone: a case report and literature review. World J Surg Oncol 2015;13:324.

2. Lai C, Teng XD. Primary enteric-type mucinous adenocarcinoma of the renal pelvis masquerading as cystic renal cell carcinoma: A case report and review of the literature. Pathol Res Pract 2016;212:842-8.

3. Hasebe M, Serizawa $S$, Chino S. On a case of papillary cystadenocarcinoma following malignant degeneration of a papillary adenoma in the kidney pelvis. Yokohama Med Bull 1960;11:491-500.

4. Shah VB, Amonkar GP, Deshpande JR, et al. Mucinous adenocarcinoma of the renal pelvis with pseudomyxoma peritonei. Indian J Pathol Microbiol 2008;51:536-7.

5. Hugen N, Brown G, Glynne-Jones R, et al. Advances in the care of patients with mucinous colorectal cancer. Nat Rev Clin Oncol 2016;13:361-9.

6. Morice P, Gouy S, Leary A. Mucinous Ovarian Carcinoma. N Engl J Med 2019;380:1256-66.

7. Shioya A, Saito K, Kurose N, et al. Primary enteric-type tubulovillous adenocarcinoma arising in the renal pelvis. Pathol Int 2018;68:388-90.

8. Patel KN, Patel NA, Gandhi S. Presentation of mucinous adenocarcinoma of renal pelvis masquerading as gross hydronephrosis: A histopathological surprise. South Asian J Cancer 2017;6:78.
9. Geramizadeh B, Khezri A, Giti R. Incidental mucinous adenocarcinoma in situ of renal pelvis presenting as severe hydronephrosis. J Nephropathol 2017;6:275-7.

10. Joseph LD, Krishnamoorthy S, Swaminathan R, et al. Mucinous Cystadenocarcinoma in a Horse Shoe Kidney Masquerading as Giant Hydronephrosis - A Case Report: Diagnostic Challenges, Lessons Learnt and Review of Literature. J Clin Diagn Res 2016;10:PD12-PD14.

11. Patel RD, Vanikar AV, Modi PR. Mucinous cystadenocarcinoma of renal pelvis presenting as pyonephrosis. Saudi J Kidney Dis Transpl 2014;25:647-50.

12. Lee HY, Jang MY, et al. Primary mucinous adenocarcinoma of the renal pelvis. Urological Science 2014;25:65-7.

13. Behzatğlu K, Boyaci C, Okçu O, et al. Mucinous urothelial carcinoma of the renal pelvis. Rare Tumors 2014;6:5485.

14. Abbas M, Kramer MW, Spieker T, et al. Primary mucinous adenocarcinoma of the renal pelvis with carcinoma in situ in the ureter. J Egypt Natl Canc Inst 2014;26:51-4.

15. Yadav R, Kataria K, Balasundaram P, et al. Mucinous cystadenocarcinoma arising in an ectopic kidney simulating a retroperitoneal dermoid cyst: a rare tumour presenting as a diagnostic dilemma. Malays J Pathol 2013;35:95-8.

16. Chang CP, Wang SS, Wen MC, et al. Mucinous adenocarcinoma of the renal pelvis masquerading as xanthogranulomatous pyelonephritis. Urology 2013;81:e40-1.

17. Chablé-Montero F, Mendoza-Ramírez S, Lavenant-Borja MI, et al. Mucinous cystadenoma of the pyelocaliceal system: a report of 3 examples and an analysis of 17 previously published cases. Ann Diagn Pathol 2013;17:239-44.

18. Sisodia SM, Khan WA, Bhavsar SP. Incidental primary papillary mucinous adenocarcinoma of the renal pelvis in a case of non-functioning kidney due to chronic pyelonephritis and pelvic calculus. Saudi J Kidney Dis Transpl 2012;23:592-3.

19. Otani M, Tsukamoto T, Serizawa H. Mucinous adenocarcinoma of the renal pelvis: A case report and review of the literature. Virchows Archiv 2012;461:S295.

20. Bharathi K, Anuradha S, Khalique A. Cytodiagnosis of mucinous cystadenocarcinoma of renal pelvis - A rare case report. International Journal of Pharma and Bio Sciences 2012;3:136-41.

21. Ye YL, Bian J, Huang YP, et al. Primary mucinous adenocarcinoma of the renal pelvis with elevated CEA and CA19-9. Urol Int 2011;87:484-8.

22. Sidharth1, Maskey P, Chalise PR, et al. Primary mucinous adenocarcinoma of the renal pelvis and ureter. Nepal Med 
Coll J 2011;13:229-30.

23. Raphael V, Sailo S, Bhuyan A, et al. Mucinous adenocarcinoma of the renal pelvis with adenocarcinoma in situ of the ureter. Urol Ann 2011;3:164-6.

24. Gulwani H, Jain A. Primary papillary mucinous adenocarcinoma of the ureter mimicking genitourinary tuberculosis. Patholog Res Int 2010;2010:738407.

25. Rao P, Pinheiro N Jr, Franco M, et al. Pseudomyxoma peritonei associated with primary mucinous borderline tumor of the renal pelvicalyceal system. Arch Pathol Lab Med 2009;133:1472-6.

26. Flores MR, Argueta VL, Ruiz MR, et al. Mucinous cystadenocarcinoma of the renal pelvis: a case report and clues to histogenesis. BMJ Case Rep 2009. doi: 10.1136/ bcr.06.2008.0307.

27. Ho CH, Lin WC, Pu YS, et al. Primary mucinous adenocarcinoma of renal pelvis with carcinoembryonic

Cite this article as: $\mathrm{Li} \mathrm{H,} \mathrm{Xie} \mathrm{F,} \mathrm{Zhao} \mathrm{C,} \mathrm{Yi} \mathrm{Z,} \mathrm{Chen} \mathrm{J,} \mathrm{Zu} \mathrm{X.}$ Primary mucinous adenocarcinoma of the renal pelvis misdiagnosed as calculous pyonephrosis: a case report and literature review. Transl Androl Urol 2020;9(2):781-788. doi: 10.21037/tau.2019.12.38 antigen production. Urology 2008;71:984.e7-8.

28. Takehara K, Nomata K, Eguchi J, et al. Mucinous adenocarcinoma of the renal pelvis associated with transitional cell carcinoma in the renal pelvis and the bladder. Int J Urol 2004;11:1016-8.

29. Mathur S, Singh MK, Rao SI, et al. Mucinous metaplasia of the renal pelvic epithelium in a case of recurrent urolithiasis and pyelonephritis. Urol Int 2004;72:355-7.

30. Kaur G, Naik VR, Rahman MN. Mucinous adenocarcinoma of the renal pelvis associated with lithiasis and chronic gout. Singapore Med J 2004;45:125-6.

31. Park S, Meng MV, Greenberg MS, et al. Muconephrosis. Urology 2002;60:344.

32. Yonekawa M, Hoshida Y, Hanai J, et al. Catheterized urine cytology of mucinous carcinoma arising in the renal pelvis. A case report. Acta Cytol 2000;44:442-4. 